\title{
DETERMINAÇÃO DE CURVAS DE LAVABILIDADE UTILIZANDO ANÁLISE DE IMAGENS DIGITAIS*
}

\section{Resumo}

Josué Mesquita de Souza Junior ${ }^{1}$ Itamar Daniel Delbem² Roberto Galery ${ }^{3}$

Luiz Claudio Monteiro Montenegro ${ }^{4}$

A concentração gravítica corresponde a uma das mais antigas e importantes formas de processamento mineral, sendo utilizada para beneficiar uma grande variedade de minérios. Neste contexto, a melhor maneira de investigar e prever resultados de processos gravíticos é a chamada "curva de lavabilidade". Estas curvas apresentam a distribuição das partículas de um dado um material segundo as suas densidades e são construídas a partir de dados experimentais obtidos nos denominados testes de flutua-afunda. Para a realização destes testes são utilizados líquidos com diferentes densidades, os quais correspondem a substâncias muito tóxicas, voláteis, de difícil manuseio e, na maioria dos casos, muito caras. Além disto, por não existirem líquidos com densidades muito elevadas, os mesmos não podem ser usados no fracionamento de sulfetos ou óxidos metálicos, o que restringe o uso das curvas de lavabilidade a materiais leves. Pensando nisto, procedeu-se ao desenvolvimento do presente trabalho, cujo objetivo é apresentar uma nova metodologia para a construção das curvas de lavabilidade a partir da análise de imagens digitais. Com esta metodologia será possível construir curvas de lavabilidade de uma maneira mais rápida e segura, além de tornar possível a aplicação desta ferramenta para minérios que possuam partículas com densidades mais elevadas.

Palavras-chave: Beneficiamento mineral; Separação gravítica; Curvas de lavabilidade; Análise de imagens digitais.

\section{DETERMINATION OF WASHABILITY CURVES USING DIGITAL IMAGE ANALISYS Abstract}

The gravity concentration corresponds to one of the oldest and most important techniques of mineral processing and it is used to process a large variety of ores. In this context, the best way of investigating and predicting results of gravity concentration processes is "washability curve". This curve shows the distribution of particles of a given material according to their densities and it is constructed from experimental data obtained under ideal conditions in so-called sink-float tests. For performing these tests dense liquids with different densities are used, which correspond to very toxic, volatile, unwieldy and, in most cases, very expensive substances. Furthermore, because there are no dense liquids with very high densities, they cannot be used in fractionation of sulfides or metal oxides, which restricts the use of washability curves to lightweight materials. With this in mind, this work was developed. The objective here is to present a new methodology for the construction of the washability curves from digital image analysis. With this method it will be possible to build washability curves in a more quickly and safely way, making possible the application of this tool for ores having particles with higher densities.

Keywords: Mineral processing; Gravity separation; Washability curves; Digital image analisys.

\footnotetext{
Engenheiro de Minas, Aluno de Mestrado PPGEM, UFMG, Belo Horizonte, MG, Brasil. Cientista da Computação, Doutor, Pesquisador, DEMIN, UFMG, Belo Horizonte, MG, Brasil..

Engenheiro de Minas, Doutor, Professor, DEMIN, UFMG, Belo Horizonte, MG, Brasil.

Engenheiro Metalurgista, Doutor, Professor, DEMIN, UFMG, Belo Horizonte, MG, Brasil.
} 


\section{INTRODUÇÃO}

A concentração gravítica pode ser definida como um processo no qual partículas de diferentes densidades, tamanhos e formas são separadas uma das outras por ação da força da gravidade atuando em conjunto com outras forças [1].

Esta é uma das mais antigas formas de processamento mineral, sendo utilizada para beneficiar uma grande variedade de minérios, variando desde metais pesados como o ouro, até materiais leves como o carvão. Para tanto, é essencial que exista uma considerável diferença de densidade entre os minerais a serem separados de modo a obter uma separação eficiente [2].

A melhor maneira de investigar e prever resultados teóricos de beneficiamento utilizando-se métodos gravíticos é a chamada curva de lavabilidade. Estas curvas apresentam a distribuição das partículas de dado um material segundo as suas densidades e são construídas a partir de dados experimentais obtidos nos denominados testes de flutua-afunda [3].

Os testes flutua-afunda consistem em passar uma amostra controladamente por uma série de líquidos de densidades decrescentes, gerando assim, dois produtos: o de menor densidade, denominado flutuado, e o de maior densidade, denominado afundado. O procedimento é repetido sucessivamente com o produto flutuado de cada etapa sendo alimentado no líquido de densidade imediatamente inferior. Os produtos resultantes, os flutuados em cada líquido e, o último produto afundado do líquido de menor densidade são, então, pesados e preparados para serem analisados quimicamente [2].

Utilizando os dados obtidos nos testes de flutua-afunda é possível construir as curvas de lavabilidade, que permitem conhecer as quantidades e as qualidades dos diferentes tipos de produtos gerados a partir de uma amostra ensaiada. Se os testes de laboratório forem bem conduzidos, os resultados obtidos serão bastante próximos do comportamento do minério nas instalações industriais, o que dará ao engenheiro uma indicação da provável eficiência do processo sob avaliação [1].

Uma desvantagem da utilização de líquidos densos é que infelizmente, além de caros, são muito tóxicos e, dessa forma, de difícil manuseio. Apresentam também, o inconveniente de não poder ser usados no fracionamento de sulfetos ou óxidos metálicos devido às altas densidades envolvidas [1]. Por este motivo o uso desta importante ferramenta se restringe atualmente ao campo de beneficiamento de carvão.

A possibilidade de conhecer o comportamento do minério em processos de separação gravítica antes mesmo de executar qualquer teste prático reduziria tanto o tempo necessário para a determinação do melhor método de beneficiamento a se utilizar, uma vez que seria possível evitar a realização de trabalhos em equipamentos inadequados, quanto os custos envolvidos no processo, através da otimização da tomada de decisão.

Tendo em vista tais fatos, o desenvolvimento de uma técnica mais simples para a construção de curvas de lavabilidade, principalmente para minérios que possuem partículas de densidades mais elevadas (como é o caso do minério de ferro) consiste em um grande avanço para a análise de operações de separação gravítica. Assim sendo, o presente trabalho tem como objetivo apresentar uma nova técnica que possibilita a construção de curvas de lavabilidade de minérios utilizando a análise de imagens digitais. Esta técnica relaciona a proporção em área de minerais presentes nas partículas analisadas em uma imagem digital com as densidades das mesmas, o que permite prever os resultados dos testes flutua-afunda sem a 
necessidade de executá-los de fato. Isto facilita a construção das curvas de lavabilidade para todos os tipos de minério, tornando a análise de processos de separação gravítica muito mais flexível e abrangente.

\section{MATERIAIS E MÉTODOS}

Para o desenvolvimento deste trabalho, foi utilizada uma amostra de minério de ferro itabirítico correspondente à alimentação de um circuito de concentração de espirais concentradoras. O minério de ferro é proveniente de um depósito localizado na região de Guanhães, no estado de Minas Gerais. A curva de lavabilidade foi construída para as frações granulométricas mais finas de $75 \mu \mathrm{m}$ a $300 \mu \mathrm{m}$ com auxílio do software Opt Lib, desenvolvido por pesquisadores da UFMG [4]. As imagens digitais foram obtidas em um microscópio ótico de luz refletida, MOLR, marca Leitz/Leica modelo Orthoplan Pol dotado de uma câmera digital Canon modelo Powershot 580. O microscópio ótico foi configurado para trabalhar com luz refletida e a câmera digital para a aquisição de imagens RGB de 24 bits com resolução espacial de 1000x1200 pixels.

O OptLib utiliza técnicas de processamento e análise digital de imagens para determinar o grau de liberação de minérios. Utilizando imagens digitais de partículas contidas em uma faixa de tamanho específica, o software permite determinar a composição areal de uma população de partículas, classifica-las por classes de composição, avaliar a liberação relativa do mineral de interesse e quantificar as diferentes fases minerais presentes na população de partículas (análise modal) [5].

Deste modo, para o desenvolvimento do trabalho, a amostra do minério de ferro foi classificada por peneiramento, sendo as frações de tamanho, [75-106] $\mu \mathrm{m},[106-150]$ $\mu \mathrm{m}$, [150-212] $\mu \mathrm{m}$ e [212-300] $\mu \mathrm{m}$ preparadas em seções polidas para serem submetidas ao processo de análise digital das imagens utilizando a metodologia desenvolvida no sistema OptLib.

Previamente ao início dos trabalhos de aquisição das imagens, os minerais presentes na amostra a ser testada foram identificados através de difração de raios$x$ utilizando-se o ICDD (International Centre for Diffraction Data) PDF-2. Os resultados mostraram que a amostra era composta majoritariamente por Hematita (densidade igual a $5,3 \mathrm{~g} / \mathrm{cm}^{3}$ ), Magnetita (densidade igual a $5,18 \mathrm{~g} / \mathrm{cm}^{3}$ ) Goetita (densidade $4,37 \mathrm{~g} / \mathrm{cm}^{3}$ ) e quartzo (densidade igual a 2,65g/cm ${ }^{3}$ ) [6].

Antes da aquisição da imagem digital e após montar as seções polidas de cada classe de tamanho no microscópio, o MOLR foi ajustado e calibrado de modo que todas as imagens digitais captadas exibissem as mesmas características de intensidade de cor para todas as amostras. Em outras palavras, isto significa que para uma fase específica, o intervalo de cores que caracteriza esta fase foi apresentado na mesma região do histograma para todas as amostras ensaiadas conforme apresentado na Figura 1. 


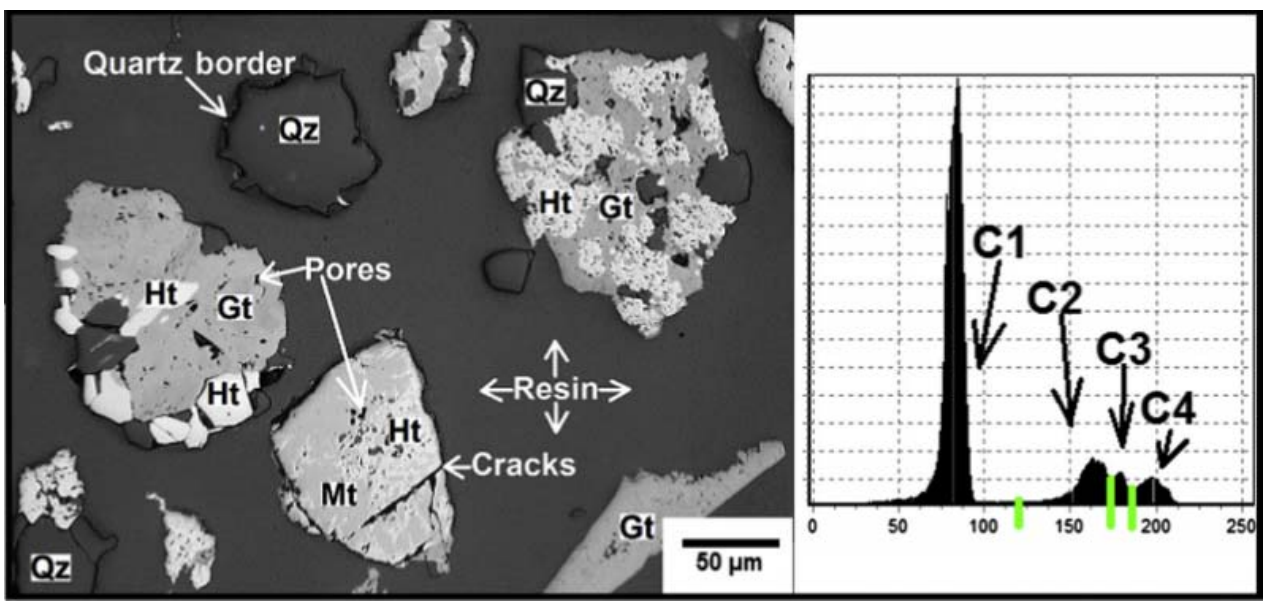

Figura 1. Imagem obtida em MOLR e seu respectivo histograma

Após a aquisição das imagens digitais, os arquivos obtidos foram submetidos a um estágio de pré-processamento constituído de: correção de fundo, sharpening, delineação e suavização. O processo completo de análise de imagens no OptLib é descrito em detalhes em [5].

A partir da análise das imagens no $O p t_{L i b}$, determinou-se as proporção em área dos minerais constituintes das amostras em cada fração granulométrica, permitindo assim, o cálculo da densidade individual de cada partícula analisada, conforme apresentado na Figura 2 e, consequentemente, a determinação das distribuições de densidade de cada faixa de tamanho.

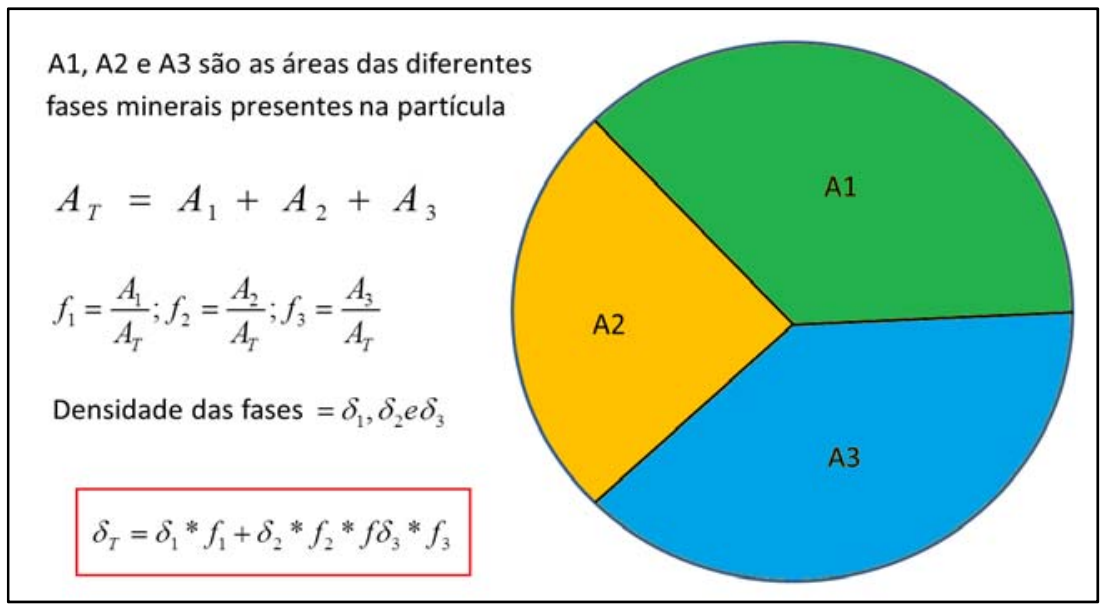

Figura 2. Metodologia de cálculo da densidade de cada partícula individual

Na construção da curva de lavabilidade, a densidade de cada partícula individual é determinada considerando as frações areais de cada fase na imagem de acordo com a Eq. 01.

$$
d_{p i}=\frac{\sum_{i=1}^{n} A_{i} \rho_{i}}{A_{T}}
$$

Onde, $d_{p i}$ é a densidade da iésima partícula, $A_{i}$ é a fração em área da fase i e $\rho_{i}$ é a densidade da fase i. $A_{T}$ é a área total das partículas em unidades de pixels. A área total da partícula considera também a área de poros, atribuindo a ela, densidade 
zero. Ponderando-se as distribuições de densidade das diferentes classes de tamanho no Opt $t_{L i b}$, foi possível construir a curva de lavabilidade da amostra de minério de ferro para as faixa granulométricas [75-106] $\mu \mathrm{m},[106-150] \mu \mathrm{m},[150-212]$ $\mu \mathrm{m}$ e [212-300] $\mu \mathrm{m}$.

\section{RESULTADOS E DISCUSSÃO}

Os resultados da análise granulométrica com a amostra de minério de ferro são apresentados na Tabela 1.

Tabela 1. Análise granulométrica da amostra de minério de ferro

\begin{tabular}{|c|c|}
\hline Malha $(\boldsymbol{\mu m})$ & Massa (\%) \\
\hline 1000 & 2,2 \\
\hline 850 & 3,1 \\
\hline 600 & 13,0 \\
\hline 425 & 17,1 \\
\hline 300 & 22,9 \\
\hline $\mathbf{2 1 2}$ & $\mathbf{1 8 , 4}$ \\
\hline $\mathbf{1 5 0}$ & $\mathbf{1 0 , 6}$ \\
\hline $\mathbf{1 0 6}$ & $\mathbf{4 , 8}$ \\
\hline $\mathbf{7 5}$ & $\mathbf{3 , 8}$ \\
\hline 38 & 2,3 \\
\hline$<38$ & 1,8 \\
\hline Total & $\mathbf{1 0 0 , 0}$ \\
\hline
\end{tabular}

Como dito anteriormente, os materiais retidos nas peneiras de $75 \mu \mathrm{m}, 106 \mu \mathrm{m}, 150$ $\mu \mathrm{m}$ e $212 \mu \mathrm{m}$ foram utilizados para a confecção de seções polidas e posterior análise no MOLR. As imagens geradas no microscópio ótico foram então processadas no software $\mathbf{O p t}_{\text {Lib }}$ tornando possível a determinação das distribuições em massa por faixa de densidade de cada fração da amostra inicial. Os resultados obtidos no Opt Lib são apresentados na Figura 2, Figura 3, Figura 4 e Figura 5 a seguir.

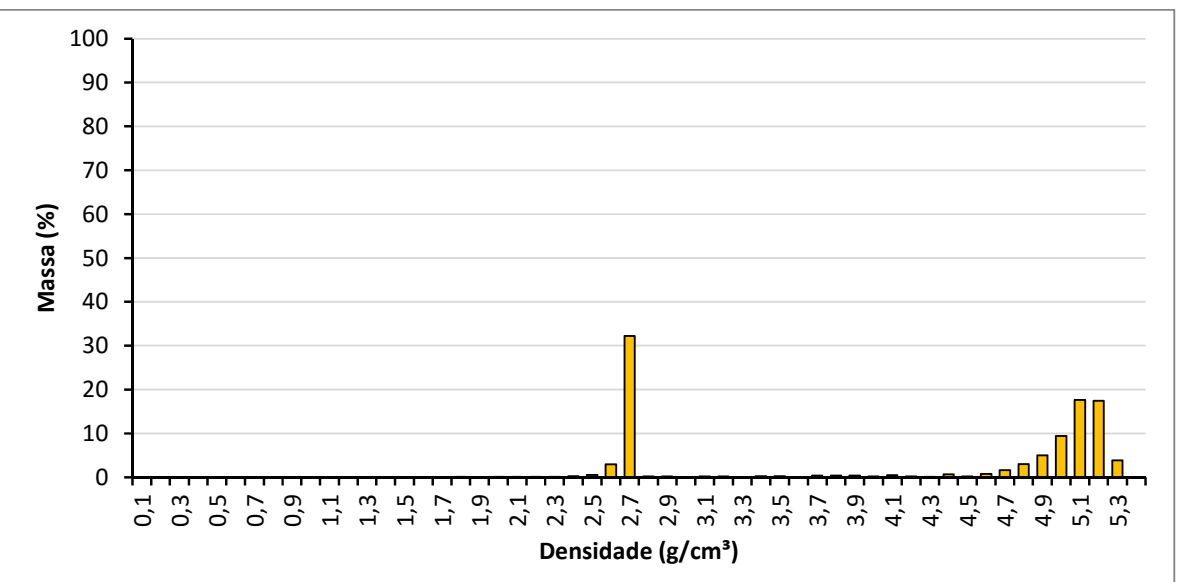

Figura 2. Distribuição em massa por faixa de densidade na fração [75-106] $\mu \mathrm{m}$ 


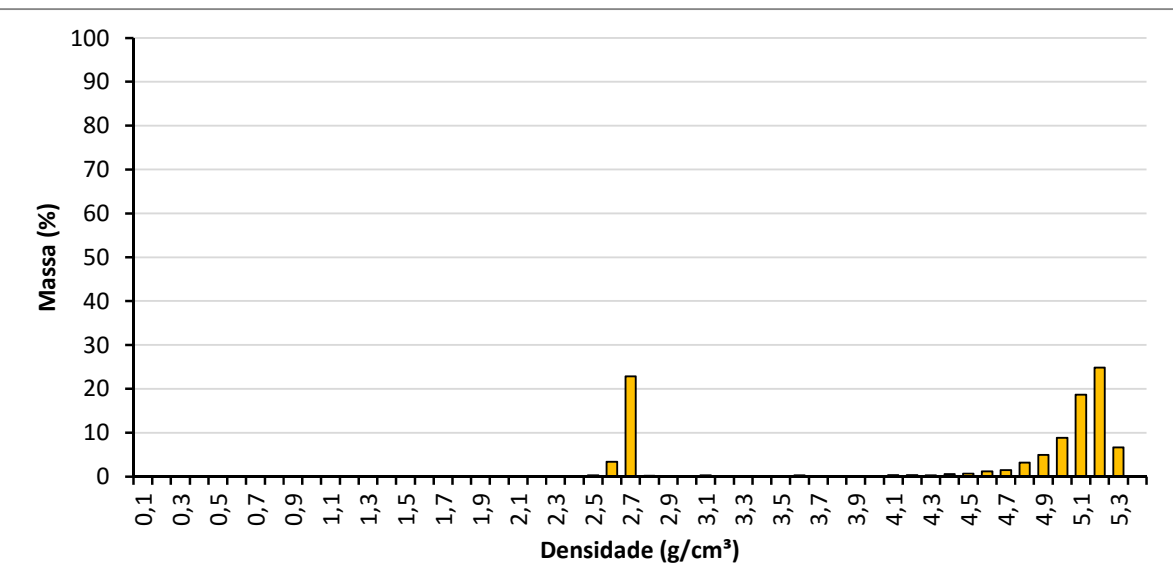

Figura 3. Distribuição em massa por faixa de densidade na fração [106-150] $\mu \mathrm{m}$

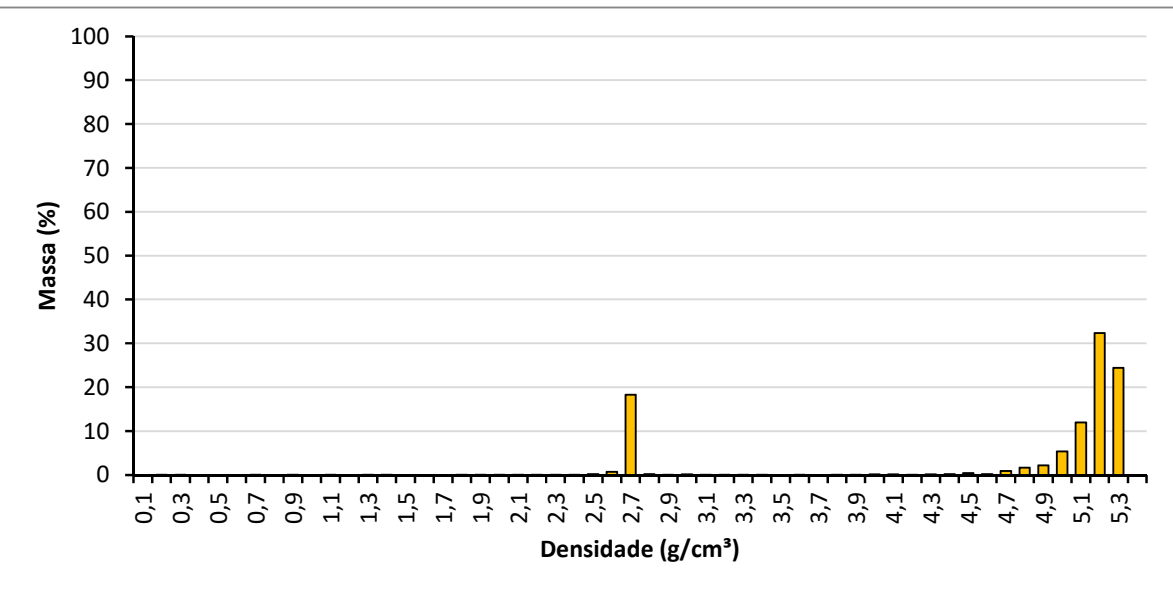

Figura 4. Distribuição em massa por faixa de densidade na fração [150-212] $\mu \mathrm{m}$

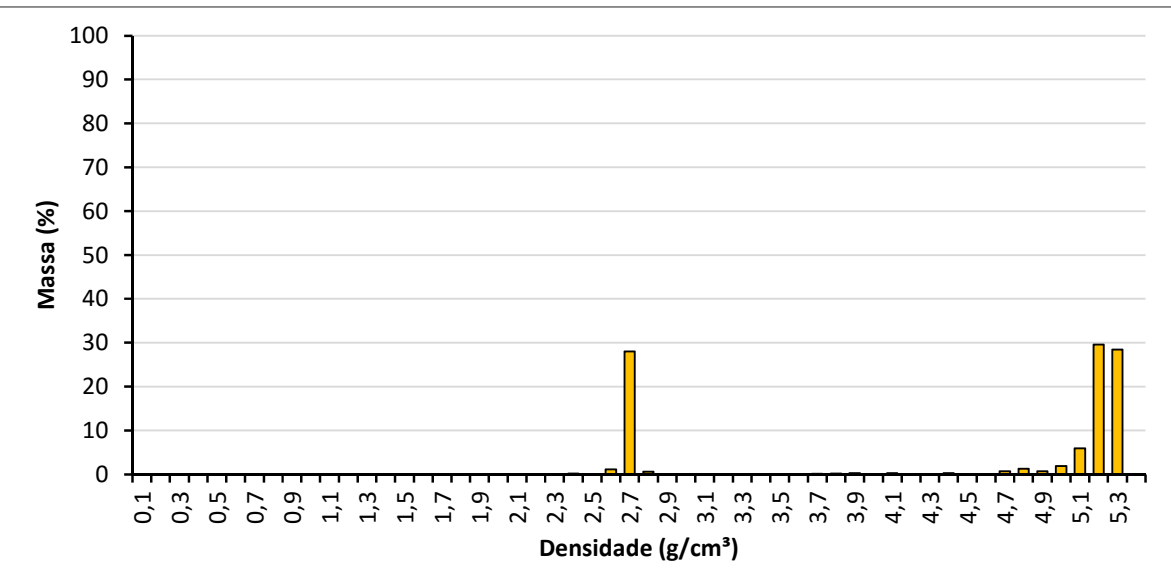

Figura 5. Distribuição em massa por faixa de densidade na fração [212-300] $\mu \mathrm{m}$

A partir da proporção retida em cada faixa granulométrica da amostra inicial e das distribuições em massa por faixa de densidade determinadas no software Opt Lib $_{\text {foi }}$ possível construir a curva de lavabilidade da amostra de minério de ferro para a fração $>75 \mu \mathrm{m}<300 \mu \mathrm{m}$, conforme apresentado na Figura 6. 


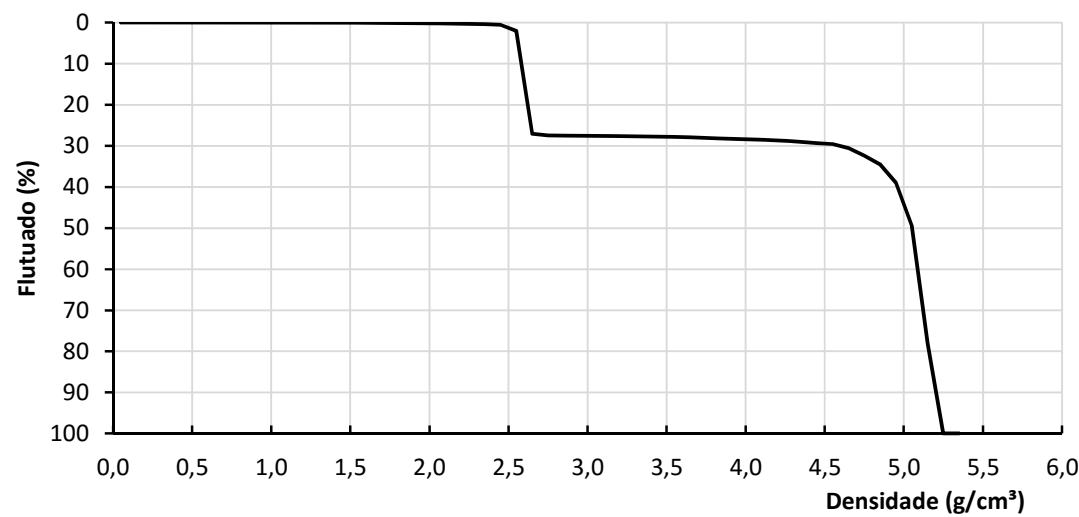

Figura 6. Curva de lavabilidade da alimentação da espiral concentradora

Como é possível observar no gráfico acima, através da curva criada é possível prever o comportamento do material sob influência de líquido densos de várias densidades sem a necessidade de executar os testes físicos. Em outras palavras, é possível estimar a quantidade de material que flutuaria caso a amostra fosse transferida para um recipiente contendo um líquido com densidade conhecida.

\section{CONCLUSÃO}

Existe um grande potencial para se construir curvas de lavabilidade através do uso de técnicas de análise digital de imagens, podendo ser um grande avanço no campo da otimização das operações de separação gravítica, principalmente para o tratamento de minérios mais densos. Um importante ponto que deve ser estudado com mais detalhes é o fato de informações em três dimensões estarem sendo analisadas em duas dimensões, o que pode causar distorções estereológicas. $O$ impacto de tal fato deve ser avaliado e mensurado de modo a tornar possível determinar a dimensão do erro advindo desta aproximação. Para tanto, se faz necessário dar continuidade a esta pesquisa focando os esforços na validação do método através de testes comparativos entre os resultados determinados através do

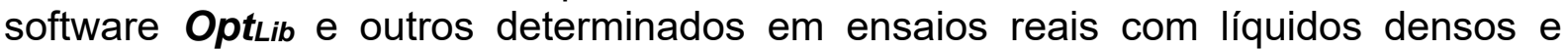
equipamentos de concentração.

\section{REFERÊNCIAS}

1 DA LUZ et al. (Ed.). Tratamento de Minérios. 5 ed. Rio de Janeiro: Cetem/MCT, 2010.

2 WILLS, B. A. Mineral Processing Technology: an introduction to the practical aspects of ore treatment and mineral recovery. 1997, Oxford.

3 MEYERS, R. A.; WALTERS, A. D. Coal preparation. Encyclopedia of science and technology, third edition, Elsevier, 2001.

4 DELBEM, I.D.; GALERY, R.; BRANDÃO, P. R. G.; PERES, A. E. C. A 2D mineral liberation analysis and phase recognition system for mineral characterization. Geomin 2011, 2nd International Seminar on Geology for the Mining Industry, Antrofagasta, 2011.

5 DELBEM, I. D. Processamento e Análise Digital de Imagens Aplicados aos Estudos de Liberação Mineral. Dissertação de mestrado, UFMG, Belo Horizonte, 2010.

6 KLEIN,C.; DUTROW, B. Manual de Ciência dos Minerais. 23a Edição. Editora Bookman, 2012. 